\section{Boston type 1 keratoprosthesis: the New York Eye and Ear experience}

\begin{abstract}
Purpose The Boston keratoprosthesis has had variable success rates in the past. However, significant modifications to design and management have recently led to successful outcomes. This study was undertaken to evaluate the outcomes of the Boston type 1 keratoprosthesis at our institution.

Methods A retrospective chart review was performed of all Boston type 1 keratoprosthesis procedures conducted at a single practice at the New York Eye and Ear Infirmary from December 2006 to August 2010. Outcome measures included visual acuity, retention rates, and complications.

Results In all, 58 eyes of 51 patients who received a Boston type 1 keratoprosthesis were included. The most common indication for the keratoprosthesis was failed penetrating keratoplasty (PK) (81.0\%; mean $2.4 \pm 1.3$ PKs
\end{abstract} per eye). Glaucoma was the most common comorbidity $(75.9 \%)$. Pre-operative best corrected visual acuity (BCVA) was $<20 / 400$ in $87.9 \%$ of eyes. At last follow-up, $43.1 \%$ of eyes

Department of Ophthalmology, Ophthalmic Consultants PC, The New York Eye and Ear Infirmary, New York, NY, USA

Correspondence: El Wu, Department of Ophthalmology, Ophthalmic Consultants PC, The New York Eye and Ear Infirmary, 310 East 14th Street, New York, NY 10003, USA Tel: + 1212505 6550; Fax: + 12129791772 E-mail: ewu@nyee.edu

Received: 19 July 2011 Accepted in revised form: 3 November 2011 Published online: 16 December 2011 had a BCVA of 20/200. Retention rate was $87.9 \%$ over an average follow-up of $21.5 \pm 11.4$ months). Complications increased with time, with $65.5 \%$ of eyes experiencing at least one event by 6 months and $75.9 \%$ by 1 year. The most common post-operative complication was retroprosthetic membrane formation $\mathbf{( 5 0 . 0 \% )}$.

Conclusions The Boston type 1 keratoprosthesis provides visual recovery for eyes with multiple PK failures or with poor prognosis for primary $\mathrm{PK}$, showing excellent retention rates. However, there is a trend towards a decline in visual acuity with time and the development of late complications, highlighting a need for longer-term studies. months (median 22 months, range 3-47
AP Patel, El Wu, DC Ritterband and JA Seedor

Eye (2012) 26, 418-425; doi:10.1038/eye.2011.325;

published online 16 December 2011

Keywords: keratoprosthesis; Boston

keratoprosthesis; Boston type 1

keratoprosthesis; artificial cornea

Introduction

Since its introduction, penetrating keratoplasty (PK) has dramatically improved visual acuity in patients with blinding corneal disease.

However, in patients undergoing repeat keratoplasty, results have been disappointing with long-term graft survival rates of only $28-55 \%$ over 5 years and $11 \%$ at 10 years. ${ }^{1-4}$ In addition, PK has shown poor visual outcomes and unacceptable failure and complication rates in high-risk conditions, such as aniridia, herpetic keratitis, severe chemical burns, and cicatrizing diseases such as ocular cicatricial pemphigoid (OCP) and StevensJohnson syndrome. ${ }^{5-8}$ Thus, the need for a new strategy for patients with poor PK prognosis has resulted in the emergence of the artificial cornea or keratoprosthesis., ${ }^{9,10}$ Because of improvements in design and postsurgical management, the use of keratoprosthesis devices and, in particular, the Boston type 1 keratoprosthesis, has increased substantially in recent years. ${ }^{11-17}$ The multicentre Boston type 1 keratoprosthesis study and several recent series have documented success of the Boston type 1 keratoprosthesis, in regard to both retention rate and visual outcomes. ${ }^{18-26}$ Traditionally advocated for use in patients with profound vision loss in both eyes, recent successful outcomes in patients with preserved vision in the fellow eye have argued for the expansion of its indication. ${ }^{24}$ However, long-term data are just emerging as many centres are beginning their artificial cornea programmes. The purpose of this study is to evaluate outcomes of the Boston type 1 keratoprosthesis at the New York 
Eye and Ear Infirmary, specifically, the visual outcomes, retention rates, and complications in comparison with more established keratoprosthesis centres.

\section{Patients and methods}

\section{Patient selection}

Patients were considered for implantation with the Boston type 1 keratoprosthesis if they are at high risk of failure with standard PK. These included patients who have had multiple failed PKs, extensive corneal vascularization or scarring, extensive limbal stem cell deficiency, or presence of conditions that do poorly following a PK such as OCP, aniridia, and severe chemical injury. Patients were deemed poor candidates for keratoprosthesis if there are comorbid conditions that would limit visual outcome including chronic retinal detachment, end-stage glaucoma, advanced macula pathology, or conditions that would lead to high risk of postoperative complications such as significant ocular surface keratinization or dryness, inadequate lid function, or poor compliance. Patients were not excluded based on the visual acuity of the fellow eye.

\section{Surgical procedure and postoperative management}

The Boston type 1 keratoprosthesis was obtained from the Massachusetts Eye and Ear Infirmary (Boston, MA, USA). The standard technique for implantation was employed as described previously. ${ }^{18}$ The diameter of the donor cornea button ranged from 8.5 to $9.0 \mathrm{~mm}$ and was $0.5 \mathrm{~mm}$ larger than recipient cornea trephination. The decision to perform concurrent glaucoma drainage implant (GDI) was based on preoperative intraocular pressure (IOP) and the amount, if known, of glaucomatous optic neuropathy. Immediately after surgery, a large diameter soft contact lens (Kontur Kontact Lens, Hercules, CA, USA) was placed to prevent desiccation and changed every 1-3 months.

Postoperatively, all patients were administered a topical steroid six times daily, and topical moxifloxacin and vancomycin $25 \mathrm{mg} / \mathrm{ml}$ four times daily. The steroid was tapered to four times daily and moxifloxacin to twice daily over 3 months, and maintained indefinitely. Vancomycin was tapered to twice daily and depending on surgeon preference, continued indefinitely in all cases or only in high-risk cases (monocular, underlying autoimmune conditions, history of previous retention failure).

\section{Data collection and analysis}

The study protocol was approved by the Institutional Review Board at The New York Eye and Ear Infirmary. A retrospective chart review was performed of all Boston type 1 keratoprosthesis procedures performed between December 2006 and August 2010. Preoperative data, such as demographics, diagnosis, comorbid conditions, and previous surgeries, were collected. Intraoperative records were reviewed for concurrent procedures performed. Postoperative data, such as visual outcomes, complications, and retention, were obtained at intervals of 1 month ( \pm 2 weeks), 6 months ( \pm 2 months), yearly ( \pm 2 months), and at last follow-up. A one-way analysis of variance was used to assess relationships between visual acuity (converted to $\log \mathrm{MAR}$ ) and nominal independent variables. A two by two crosstab using Fisher's exact test was used to compare frequency differences between various patient subgroups. A two-sided $P$-value of $<0.05$ was considered statistically significant. Statistical analysis was performed using SPSS software version 19.0 (IBM, Armonk, NY, USA).

\section{Results}

\section{Preoperative data}

From December 2006 to August 2010, 58 eyes in 51 patients (35 females and 16 males) underwent Boston type 1 keratoprosthesis ( 7 patients had implants in both eyes). Mean age at the time of surgery was 62 (range 16-91). Preoperative visual acuity ranged from $20 / 200$ to light perception, the median being count fingers, with $87.9 \%$ of eyes having best corrected visual acuity (BCVA) $<20 / 400$. Visual acuity of the fellow eye ranged from $20 / 20$ to no light perception $(20 / 20-20 / 100 n=28$, 20/200-20/400 $n=9$, count fingers-hand motion $n=9$, light perception $n=3$, no light perception $n=7$ ). In two patients, the fellow eye had been previously enucleated.

The main indication was prior failed grafts $(47 / 58$ eyes, $81.0 \%$, mean $2.4 \pm 1.3$ prior PKs, range $1-6$ ) (Table 1). In all, 4 out of the 47 eyes additionally had failed AlphaCor keratoprosthesis following multiple failed PKs. The most common diagnoses for the initial PK were infectious keratitis, endothelial failure following multiple surgeries, pseudophakic and aphakic bullous keratopathy. In 11 eyes (19.0\%), the keratoprosthesis was performed as the primary surgery for aniridia $(n=3)$, endothelial failure following surgery $(n=3), \mathrm{OCP}(n=2)$, pseudophakic bullous keratopathy with stem cell deficiency $(n=1)$, infectious keratitis with history of corneal perforation $(n=1)$, and chemical injury $(n=1)$.

Glaucoma was the most common comorbidity, present in $75.9 \%(44 / 58)$ of eyes. In all, 26 eyes $(44.8 \%)$ had prior GDI placement and 11 eyes (19.0\%) had prior trabeculectomy. Other preexisting comorbid conditions included previous retinal detachment repair (12.1\%), macular pathology (10.3\%), uveitis (6.9\%), and hypotony $(3.4 \%)$ (Table 2). 
Table 1 Preoperative diagnosis

\begin{tabular}{|c|c|}
\hline Preoperative diagnosis & $\begin{array}{l}\text { No. of } \\
\text { eyes }\end{array}$ \\
\hline \multicolumn{2}{|l|}{ No prior corneal transplant $(\mathrm{n}=11)$} \\
\hline Aniridia & 3 \\
\hline Failed cornea after multiple surgeries & 3 \\
\hline Ocular cicatricial pemphigoid & 2 \\
\hline $\begin{array}{l}\text { Pseudophakic bullous keratopathy/limbal stem cell } \\
\text { deficiency }^{\mathrm{a}}\end{array}$ & 1 \\
\hline $\begin{array}{l}\text { Infectious keratitis/post radiation following } \\
\text { sebaceous cell carcinoma }\end{array}$ & 1 \\
\hline Chemical injury & 1 \\
\hline \multicolumn{2}{|l|}{ Failed corneal transplant $(\mathrm{n}=47)$} \\
\hline Infectious keratitis & 10 \\
\hline Failed cornea after multiple surgeries & 9 \\
\hline Pseudophakic/aphakic bullous keratopathy & 9 \\
\hline Fuch's endothelial dystrophy & 5 \\
\hline Aniridia & 3 \\
\hline Keratoconus & 3 \\
\hline Chemical injury & 2 \\
\hline Ocular cicatricial pemphigoid & 1 \\
\hline Peters anomaly & 1 \\
\hline Iridocorneal endothelial syndrome & 1 \\
\hline Pellucid marginal degeneration & 1 \\
\hline Lattice dystrophy & 1 \\
\hline Trauma & 1 \\
\hline
\end{tabular}

${ }^{a}$ This patient had a prior total conjunctival flap and was not a candidate for a PK with stem cell transplant and systemic immunosuppression due to a prior gastric malignancy.

Table 2 Comorbid conditions

\begin{tabular}{lc}
\hline Condition & No. of eyes (\%) \\
\hline Glaucoma & $44(75.9)$ \\
Glaucoma drainage implant & $26(44.8)$ \\
Trabeculectomy & $11(19.0)$ \\
Prior retinal detachment with repair & $7(12.1)$ \\
Macular pathology & \\
Uveitis & $6(10.3)$ \\
Hypotony & $4(6.9)$ \\
\hline
\end{tabular}

${ }^{a}$ Epiretinal membrane, macular hole, macular chorioretinal scar, age-related macular degeneration.

\section{Intraoperative data}

The pseudophakic Boston type 1 keratoprosthesis was implanted in $53.4 \%$ of eyes and the aphakic keratoprosthesis in $46.6 \%$. Eight of the implants had a backplate diameter of $8.5 \mathrm{~mm}$ and fifty had a backplate diameter of $7.0 \mathrm{~mm}$. Two implants had the titanium backplates and the remaining PMMA backplates. All the 58 eyes had a titanium locking ring.

Thirty eyes had at least one other concurrent procedure. GDI placement was performed in $31.0 \%$ of eyes (18/58), 17 of which had prior history of glaucoma and 10 out of these 17 having previous glaucoma surgery.
Overall, $38.6 \%$ of glaucomatous eyes (17/44) had a GDI placed intraoperatively. There was a statistically significant difference with preoperative IOP in glaucomatous patients with concurrent GDI placement and in those without $(21.7 \pm 8.5$ versus $14.2 \pm 5.9 \mathrm{~mm} \mathrm{Hg}$, $P=0.001$ ).

Other intraoperative procedures included vitrectomy (35.1\%), IOL removal (24.1\%), and cataract extraction $(8.6 \%)$.

\section{Postoperative data}

Visual outcomes

Mean follow-up was 21.5 \pm 11.4 months (median 22 months, range 3-47 months). In all, 7 eyes had 3-6 months of follow-up, 9 eyes with 7-12 months, 17 eyes with 13-24 months, 21 eyes with 25-36 months, and 4 eyes with $37-48$ months. At the last follow-up, $43.1 \%$ of eyes attained BCVA $\geq 20 / 200$. By follow-up, the percentage of eyes with BCVA $\geq 20 / 200$ showed a decreasing trend with $74.5 \%(35 / 47)$ at 1 year, $50.0 \%(16 / 32)$ at 2 years, and $36.3 \%(4 / 11)$ at 3 years. Visual change following keratoprosthesis by presenting acuity is summarized in Table 3 . At the last follow-up, BCVA improved in $55.2 \%$ of eyes $(32 / 58)$, remained the same in $27.6 \%$ of eyes $(16 / 58)$, and worsened in $17.2 \%$ of eyes $(10 / 58)$ because of postoperative complications. Four were retention failures described in Table 5 (patients $1,2,3$, and 6). In the remaining six eyes, visual deterioration occurred because of glaucoma in two patients (vision CF to HM and HM to LP), glaucoma and recurrent retroprosthetic membrane (RPM) in two patients (vision 20/400 to HM and LP to NLP), glaucoma and vitreous haemorrhage in one patient (vision 20/400 to LP), and retinal detachment in one patient (vision CF to $\mathrm{HM}$ ). Evaluating time at which BCVA was reached for each eye showed that $53.4 \%(31 / 58)$ achieved their BCVA by 1 month postoperatively, and an additional $25.9 \%$ by 6 months.

Subgroup analysis of visual outcomes was performed by diagnosis, history of prior PK versus no PK, and history of preexisting comorbidity (glaucoma, retinal detachment, and macular pathology). Eyes with Fuch's dystrophy had a statistically significant improvement in visual acuity at last visit $(P=0.03)$. Eyes with OCP had worsening of visual acuity $(P=0.03)$. History of prior $P K$, glaucoma, and macular pathology did not significantly affect visual outcome, whereas prior retinal detachment was associated with less improvement in visual acuity $(P=0.04)$.

\section{Complications}

Overall, $77.6 \%$ of eyes (45/58) developed at least one complication following surgery, $25.9 \%$ of eyes with two 
Table 3 Last-visit visual acuity based on preoperative visual acuity

\begin{tabular}{|c|c|c|c|c|c|}
\hline \multirow[t]{2}{*}{ Postoperative visual acuity } & \multicolumn{5}{|c|}{ Preoperative visual acuity } \\
\hline & $L P(\mathrm{n}=10)$ & $H M(\mathrm{n}=19)$ & $C F(\mathrm{n}=22)$ & $20 / 400(n=6)$ & $20 / 200(\mathrm{n}=1)$ \\
\hline NLP & $1(10 \%)$ & $1(5.3 \%)$ & $2(9.1 \%)$ & 0 & 0 \\
\hline LP & $5(50 \%)$ & $1(5.3 \%)$ & 0 & $1(16.7 \%)$ & 0 \\
\hline HM & $2(20 \%)$ & $5(26.3 \%)$ & $2(9.1 \%)$ & $2(33.3 \%)$ & 0 \\
\hline $\mathrm{CF}$ & 0 & $1(5.3 \%)$ & $4(18.2 \%)$ & 0 & 0 \\
\hline $20 / 400$ & $1(1 \%)$ & $3(15.8 \%)$ & $1(4.5 \%)$ & $1(16.7 \%)$ & 0 \\
\hline $20 / 200$ & 0 & $2(10.5 \%)$ & $3(13.6 \%)$ & 0 & $1(100 \%)$ \\
\hline $20 / 50-20 / 100$ & 0 & $4(21.1 \%)$ & $8(36.4 \%)$ & $2(33.3 \%)$ & 0 \\
\hline$>20 / 50$ & $1(1 \%)$ & $2(10.5 \%)$ & $2(9.1 \%)$ & 0 & 0 \\
\hline
\end{tabular}

Abbreviations: $\mathrm{CF}$, count fingers; HM, hand motion; LP, light perception; NLP, no light perception. Improved visual acuity below the weighted line.

Table 4 Postoperative complications and procedures

\begin{tabular}{lc}
\hline Complications & No. of cases (\%) \\
\hline Retroprosthetic membrane & $29(50.0)$ \\
Increased intraocular pressure & $15(25.9)$ \\
Retinal detachment & $6(10.3)$ \\
Cystoid macular oedema & $5(8.6)$ \\
Vitreous haemorrhage & $3(5.2)$ \\
Corneal necrosis & $2(3.4)$ \\
Prosthesis extrusion & $2(3.4)$ \\
Persistent epithelial defect & $2(3.4)$ \\
Sterile vitritis & $1(1.7)$ \\
Choroidal detachment & $1(1.7)$ \\
Endophthalmitis & $1(1.7)$ \\
& \\
Procedures & No. of occurrences \\
\hline YAG membranotomy & 17 \\
Keratoprosthesis explant & 7 \\
Retinal detachment repair & 4 \\
Surgical membranectomy & 2 \\
Glaucoma drainage implant & 2 \\
Transcleral cyclophotocoagulation & 1 \\
Epiretinal membrane peel & 1 \\
Choroidal drainage & 1 \\
Intraocular lens removal (for RPM) & 1 \\
\hline
\end{tabular}

Abbreviation: YAG, yttrium-aluminum-garnet.

complications, and $5.2 \%$ of eyes with three complications (Table 4). Incidence of postoperative complications increased with follow-up, with $19.0 \%$ of eyes experiencing at least one adverse event by 1 month, $65.5 \%$ by 6 months, and $75.9 \%$ by 1 year. RPM formation was the most common, occurring in $50.0 \%$ of eyes (29/58), 19 of which were visually significant. The majority were successfully treated with yttriumaluminium-garnet membranotomy with two eyes requiring surgical membranectomy. Recurrent RPM developed in 11 eyes, 1 of which required keratoprosthesis exchange. Eyes with a history of a retinal detachment were more likely to develop RPM $(P=0.048)$. Initial diagnosis, history of glaucoma, and performance of concurrent operative procedures did not significantly increase RPM formation.

Increased IOP as determined by tactile tension occurred in $25.9 \%$ of eyes $(15 / 58)$, mostly in eyes with prior history of glaucoma (accounts for 11/15 eyes and 10 had prior GDI). Out of the 18 eyes that underwent GDI concurrently with the keratoprosthesis, only 2 (11.1\%) developed elevated IOP postoperatively. All cases were initially managed with topical glaucoma medication with 1 patient eventually requiring transscleral cyclophotocoagulation. Two eyes subsequently underwent a GDI at a later date. No complications related to GDI placed at the time of keratoprosthesis or subsequently were observed. In these 15 eyes that developed elevated IOP, visual acuity at last visit was better than prior to keratoprosthesis in 8 eyes, remained the same in 4 eyes, and worsened in 3 eyes.

Retinal detachment occurred in six eyes (10.3\%), one of which had prior history of retinal detachment. The underlying diagnoses in these patients were aniridia in three eyes, OCP, infectious keratitis, and failed cornea after multiple surgeries. Two detachments occurred in eyes with retention failure following the keratoprosthesis explantation. Successful repair was initially achieved in four eyes with the remaining two eyes deemed inoperable due to the extensive detachment or proliferative vitreoretinopathy. Of the four that were successfully repaired initially, two developed recurrent detachments that were inoperable. In all six patients, visual acuity at last visit was equal or worse than acuity before keratoprosthesis.

Cystoid macular oedema occurred in five eyes (8.6\%). In two, cystoid macular oedema resolved following 
Table 5 Cases of retention failures

\begin{tabular}{|c|c|c|c|c|c|c|c|}
\hline $\begin{array}{l}\text { Patient } \\
\text { no. }\end{array}$ & Reason for removal & $\begin{array}{c}\text { Onset } \\
\text { (month) }\end{array}$ & Original diagnosis & $\begin{array}{l}\text { Preoperative } \\
B C V A\end{array}$ & Management & Complications & $\begin{array}{l}\text { Final } \\
B C V A\end{array}$ \\
\hline 1 & $\begin{array}{l}\text { Persistent RPM unable to } \\
\text { be removed surgically }\end{array}$ & 8 & $\begin{array}{l}\text { Ocular cicatricial } \\
\text { pemphigoid }\end{array}$ & $\mathrm{HM}$ & Repeat kpro & $\begin{array}{l}\text { Increased IOP, inoperable } \\
\text { retinal detachment }\end{array}$ & NLP \\
\hline 2 & $\begin{array}{l}\text { Retinal detachment and } \\
\text { persistent RPM }\end{array}$ & 8 & Aniridia & $20 / 400$ & Repeat kpro & $\begin{array}{l}\text { Recurrent RPM, retinal } \\
\text { detachment }\end{array}$ & $\mathrm{HM}$ \\
\hline 3 & Prosthesis extrusion & 9 & Aniridia & CF & PK & $\begin{array}{l}\text { Inoperable retinal } \\
\text { detachment }\end{array}$ & NLP \\
\hline 4 & $\begin{array}{l}\text { Corneal necrosis (Candida } \\
\text { parapsilosis) }\end{array}$ & 4 & Infectious keratitis & LP & Repeat kpro & Prosthesis extrusion & $\mathrm{HM}$ \\
\hline 5 & $\begin{array}{l}\text { Corneal necrosis (Bacillus } \\
\text { cereus) }\end{array}$ & 2 & $\begin{array}{l}\text { Fuch's endothelial } \\
\text { dystrophy }\end{array}$ & $\mathrm{HM}$ & PK & None & $20 / 200$ \\
\hline 6 & $\begin{array}{l}\text { Endophthalmitis } \\
\text { (Sphingomonas paucimobilis) }\end{array}$ & 3 & $\begin{array}{l}\text { Pellucid marginal } \\
\text { degeneration }\end{array}$ & $\mathrm{CF}$ & Enucleation & None & NLP \\
\hline 7 & Prosthesis extrusion & 6 & Chemical injury & LP & Repeat kpro & $\begin{array}{l}\text { Choroidal haemorrhage, } \\
\text { prosthesis extrusion }\end{array}$ & LP \\
\hline
\end{tabular}

Abbreviations: CF, count fingers; HM, hand motion; LP, light perception; NLP, no light perception.

topical treatment and attained BCVA of 20/60 and 20/70. The remaining three eyes with persistent cystoid macular oedema at 6,7 , and 39 months had final acuity of 20/400, 20/100, and count finger, respectively.

Significant corneal necrosis developed in two eyes (patients 4 and 5 in Table 5), both due to microbial keratitis (Candida parapsilosis and Bacillus cereus). Both patients were compliant with topical antibiotics and contact lens wear. Patient 4 with Candida had a history of exposure keratopathy related to HIV facial wasting. One eye was treated with repeat keratoprosthesis implantation (final acuity HM), whereas the other eye underwent a PK (final acuity 20/200).

Endophthalmitis occurred in one patient at 1 month (patient 6 in Table 5) with Sphingomonas paucimobilis in the setting of noncompliance with topical antibiotics. Vision deteriorated to no light perception despite treatment with intravitreal and topical fortified antibiotics. The eye eventually required enucleation because of severe pain.

Overall, 37 surgical procedures in 28 eyes (48.3\%) were performed to manage postoperative complications, the most common being yttrium-aluminium-garnet membranectomy $(n=17)$, followed by keratoprosthesis explantation $(n=7)$, and retinal detachment repair $(n=4)$ (Table 4).

\section{Retention}

Retention rate was $87.9 \%(51 / 58)$ over an average followup of $21.5 \pm 11.4$ months (range 3-47 months). All retention failures occurred in the first year and were due to prosthesis extrusion $(n=2)$, infectious corneal necrosis $(n=2)$, endophthalmitis $(n=1)$, recurrent RPM unamenable to membrane removal $(n=1)$, and retinal detachment with RPM $(n=1)$ (Table 5$)$. Four eyes underwent repeat keratoprosthesis, two eyes had tectonic PK, and the endophthalmitis eye was enucleated. In all except the two cases of corneal necrosis following infectious keratitis, visual acuity deteriorated after experiencing retention failure. Retention failures accounted for three out of the four cases of final NLP visual acuity in the series.

\section{Discussion}

In our clinical experience with the Boston type 1 keratoprosthesis, a significant number of patients attained a favourable outcome with $55 \%$ of eyes experiencing improved visual acuity, comparable to the $57 \%$ reported in the multicentre Boston keratoprosthesis study. ${ }^{18}$ In these patients who generally have poor preoperative vision, a significant number were able to achieve ambulatory vision. Although only one patient had 20/200 vision preoperatively and none better, $43 \%$ achieved 20/200 or better at their last visit following keratoprosthesis implantation. In addition, compared with standard PK, the Boston keratoprosthesis provided relatively rapid visual recovery, with $53 \%$ of our patients attaining their BCVA by the 1-month postoperative visit and $79 \%$ by 6 months. Dunlap et al ${ }^{26}$ also demonstrated rapid visual improvement with $56 \%$ of their patients achieving their BCVA at month 1 . Several recent keratoprosthesis series report visual outcomes by time points. Our percentage of patients with 20/200 vision or better at 1 year $(75 \%)$ was comparable to that of other series $\left(75 \%\right.$ in Bradley et $a l^{23}, 82 \%$ in Aldave et $a l^{24}$, and $77 \%$ in Chew et $a l^{25}$ ). However, subgroup analysis by time in our series shows that this percentage decreases to $50 \%$ by 2 years and $36 \%$ by 3 years, which may reflect development of late complications or may be due to significantly smaller sample sizes at the longer follow-up. The deterioration of vision following 
keratoprosthesis that occurred in $17 \%$ of eyes was due in part to progression of the underlying disease, such as glaucoma, and from post-operative development of RPM or retinal detachment.

The main indication of keratoprosthesis implantation remains prior failed corneal transplant. Our keratoprosthesis survival rate of $87.9 \%$ at a mean follow-up of 21.5 months compares favourably with that of repeat PK (63\% survival at 1 year with first or subsequent regraft with rapid decline thereafter to only $28 \%$ for first regraft and $20 \%$ for subsequent regraft at 54 months). ${ }^{3}$ Because keratoprosthesis patients are specifically chosen because of the belief that repeat PK would result in graft failure, it could be inferred that repeat keratoplasty in this high-risk group would have worse results than the survival results quoted above. Our survival rate is comparable to the rates in recent keratoprosthesis series (91.6\% with an average follow-up of 13 months in the multicentre Boston type 1

keratoprosthesis study, ${ }^{27} 83.3 \%$ at an average follow-up of 19 months in Bradley et al, ${ }^{23} 84 \%$ at an average follow-up of 17 months in Aldave et $a l^{24}$ ). Longer follow-up for keratoprosthesis is required to assess whether the superior survival function compared with repeat PK will be maintained.

One barrier to keratoprosthesis implantation remains the concern over the high risk of postoperative complications. In our series, a significant percentage of patients $(78 \%)$ did develop at least one adverse event. However, complications that resulted in visual loss (from glaucoma, retinal detachment, or RPM) occurred in a significantly lower number $(17 \%)$. The most common postoperative complication was RPM formation, developing in $50 \%$ of the eyes. Other series also support the common occurrence of RPM (14-65\%). ${ }^{23-26}$ It has been suggested that inflammation as well as performance of secondary operative procedures at the time of keratoprosthesis implantation is related to the development of RPM, ${ }^{24,28}$ however, in our study we did not find an association with secondary procedures. Despite the high prevalence of RPM, most were successfully treated with yttrium-aluminiumgarnet membranotomy echoing the need for early detection and treatment. . $^{23-25,29}$ Todani et al ${ }^{30}$ in a multicentre study reported that eyes with titanium backplates had a statistically significant less chance of inducing RPMs than its PMMA counterparts. Since the titanium plates were introduced more recently, our series had only two titanium plates so we cannot comment on this observation. Ament et $a l^{31}$ in an in vitro cell model using human corneal-limbal cell cultures noted decreased cell death with cell/titanium contact compared with cell/PMMA contact. It remains to be determined whether the titanium backplate will cause a decrease in RPM formation over the long term.

A commonly reported challenge after keratoprosthesis implantation is accurate assessment of IOP, a particular concern as glaucoma is a common comorbidity in these patients. Consistent with other recent series, our cohort had a high percentage of patients with preexisting glaucoma (76\% compared with $66 \%$ in Bradley et al, ${ }^{23}$ $75 \%$ in Aldave et $a l^{24}$ and $73 \%$ in Chew et $a l^{25}$ ). Despite this high prevalence, the percentage of patients who developed elevated IOP following keratoprosthesis was $26 \%$, within the range from other series $(14 \%$ in multicentre Boston keratoprosthesis study, ${ }^{18} 18 \%$ in Aldave et $a l^{24}$ and $38 \%$ in Chew et $a l^{25}$ ) and occurred mostly in patients with prior diagnosis of glaucoma. Most were successfully managed with medication with only three eyes requiring secondary glaucoma surgery. However, it should be noted that the concurrent placement of a GDI in $31 \%$ of eyes precludes the determination of the true rate of elevated IOP following keratoprosthesis placement (likely leading to an underestimation). Given the difficulty of monitoring IOP and the elevation that often occurs after keratoprosthesis implantation, some authors have supported the implantation of a GDI in patients with a keratoprosthesis. ${ }^{32}$ In our series, the decision to place a GDI was based on the extent of glaucomatous damage and the preoperative IOP. Although concomitant GDI placement at the time of PK can lead to graft failure, ${ }^{33,34}$ this is not a concern during keratoprosthesis implantation. In our cohort, no complications were directly attributed to GDI placement and no GDI erosion was noted. Furthermore, GDI seemed effective in decreasing glaucoma progression, which was noted only in 2 out of 18 patients who had concurrent GDI. As such, the authors support the concurrent placement of GDI in patients with uncontrolled or borderline IOP.

Retinal detachment occurred in $10.3 \%$ of eyes $(3.5 \%$ in multicentre Boston type I keratoprosthesis study, ${ }^{18} 7 \%$ in Aldave $e t a l^{24}$ and $4.8 \%$ in Dunlap et $a l^{26}$ ). Three of the six cases occurred in patients with aniridia, with one in the setting of a prior retinal detachment. All cases of retinal detachment had poor visual outcome, which has also been found in another report of retinal detachment in keratoprosthesis patients. ${ }^{35}$

Keratolysis occurred in two eyes in the setting of microbial keratitis. In addition to prophylactic antibiotics, use of a large diameter BCL is encouraged to decrease risk of desiccation and secondary infection. Both patients with microbial keratitis were able to wear their contact lens and were compliant with antibiotics. However, one patient had a history of exposure keratopathy due to HIV-related facial wasting and had 
received Sculptra (injectable poly-L-lactic acid) and lid-tightening surgery. He remained with mild exposure and developed corneal melt due to Candida, which was unresponsive to voriconazole. A crescenteric amniotic membrane graft as described by Tay $e t a l^{36}$ was placed, but it was unable to control the keratolysis and the implant eventually extruded. C. parapsilosis has been reported in previous reviews as a common organism found in culture-positive fungal infections. ${ }^{24,37}$ Once corneal necrosis occurs, it can be difficult to manage medically. Both keratitis patients required exchange of the keratoprosthesis, however, each still achieved improved postoperative visual acuity.

Since the routine use of maintenance prophylactic antibiotics, the incidence of endophthalmitis has decreased from $12 \%$ in earlier studies to negligible in recent series. ${ }^{15,16}$ In our series, one eye developed endophthalmitis with S. paucimobilis. However, this was in the setting of noncompliance with prophylactic antibiotics. Our low rate of endophthalmitis supports other recent series in which either endophthalmitis did not occur or occurred mostly in patients who were noncompliant. ${ }^{23-25}$

In our series, we found an association of OCP with poor visual outcomes. We caution against the use of the Boston type 1 keratoprosthesis in eyes with cicatricial diseases, such as OCP, or in moderate ocular surface dysfunction, such as in the case of our post-radiation patient secondary to sebaceous cell carcinoma who, despite a perceived adequate tear film, had significant surface keratinization and vascularity. If the decision is made to proceed with an artificial cornea in these patients, consideration of an osteo-odonto keratoprosthesis device should be entertained using a program that has established success with these devices.

\section{Conclusions}

Our experience at The New York Eye and Ear Infirmary with the Boston type 1 keratoprosthesis is comparable to the other recent series and to the initial multicentre Boston keratoprosthesis study in terms of visual improvement, high retention rate, and complication profile. In high-risk patients whom a standard PK offers little chance of visual recovery with unacceptable high risk of failure, the Boston type 1 keratoprosthesis is a viable option. However, several challenges unique to the keratoprosthesis remain, including the accurate assessment of glaucoma status, maintenance of the opticcornea interface, and the formation of RPM. In addition, the trend of a decline in visual acuity with time warrants longer studies with particular regard to late complications.
Summary

What was known before

- The Boston type 1 keratoprosthesis has had recent successful outcomes in the short term. One barrier to more widespread use of keratoprosthesis remains high rates of complications such as glaucoma progression and retroprosthetic membrane formation.

What this study adds

- The Boston type 1 keratoprosthesis is a viable option for patients with poor prognosis for penetrating keratoplasty with favorable visual outcomes and high retention rates. However, there is a trend towards a decline in visual acuity with time with development of late complications. This study highlights the need for longer-term studies. This study also recommends early and aggressive treatment of complications, such as retroprosthetic membranes and prophylactic measures, to prevent glaucoma progression.

\section{Conflict of interest}

The authors declare no conflict of interest.

\section{References}

1 Beckingsale P, Mavrikakis I, Al-Yousuf N, Mavrikakis E, Daya SM. Penetrating keratoplasty: outcomes from a corneal unit compared to national data. Br J Ophthalmol 2006; 90: 728-731.

2 Muraine M, Sanchez C, Watt L, Retout A, Brasseur G. Longterm results of penetrating keratoplasty: a 10-year-plus retrospective study. Graefes Arch Clin Exp Ophthalmol 2003; 241: $571-576$.

3 Bersudsky V, Blum-Hareuveni T, Rehany U, Rumelt S. The profile of repeated corneal transplantation. Ophthalmology 2001; 108: 461-469.

4 Ma JJ, Graney JM, Dohlman CH. Repeat penetrating keratoplasty versus the Boston keratoprosthesis in graft failure. Int Ophthalmol Clin 2005; 45: 49-59.

5 Yaghouti F, Nouri M, Abad JC, Power WJ, Doane MG, Dohlman $\mathrm{CH}$. Keratoprosthesis: preoperative prognostic categories. Cornea 2001; 20: 19-23.

6 Ing JJ, Ing HH, Nelson LR, Hodge DO, Bourne WM. Ten-year postoperative results of penetrating keratoplasty. Ophthalmology 1998; 105: 1855-1865.

7 Tugal-Tutkun I, Akova YA, Foster CS. Penetrating keratoplasty in cicatrizing conjunctival diseases. Ophthalmology 1995; 102: 576-585.

8 Kremer I, Rajpal RK, Rapuano CJ, Cohen EJ, Laibson PR. Results of penetrating keratoplasty in aniridia. Am J Ophthalmol 1993; 115: 317-320.

9 Barber JC. Keratoprosthesis: past and present. Int Ophthalmol Clin 1988; 28: 103-109.

10 Hicks CR, Fotton JH, Chirila TV, Crawford GJ, Constable IJ. Keratoprosthesis: advancing toward a true artificial cornea. Surv Ophthalmol 1997; 42: 175-189.

11 Doane MG, Dohlman CH, Bearse G. Fabrication of keratoprosthesis. Cornea 1996; 15: 179-184.

12 Harissi-Dagher M, Khan BF, Schaumberg DA, Dohlman CH. Importance of nutrition to corneal grafts when used as a carrier of the Boston keratoprosthesis. Cornea 2007; 26: 564-568. 
13 Dohlman $\mathrm{CH}$, Dudenhoefer EJ, Khan BF, Morneault S. Protection of the ocular surface after keratoprosthesis surgery: the role of soft contact lenses. CLAO J 2002; 28: 72-74.

14 Harissi-Dagher M, Beyer J, Dohlman $\mathrm{CH}$. The role of soft contact lenses as an adjunct to the Boston keratoprosthesis. Int Ophthalmol Clin 2008; 48: 43-51.

15 Khan BF, Harissi-Dagher M, Khan DM, Dohlman CH. Advances in Boston keratoprosthesis: enhancing retention and prevention of infection and inflammation. Int Ophthalmol Clin 2007; 47: 61-71.

16 Nouri M, Terada H, Alfonso EC, Foster CS, Durand ML, Dohlman $\mathrm{CH}$. Endophthalmitis after keratoprosthesis: incidence, bacterial causes, and risk factors. Arch Ophthalmol 2001; 119: 484-489.

17 Durand ML, Dohlman CH. Successful prevention of bacterial endophthalmitis in eyes with the Boston keratoprosthesis. Cornea 2009; 28: 896-901.

18 Zerbe BL, Belin MW, Ciolino JB. Results from the multicentre Boston type I keratoprosthesis study. Ophthalmology 2006; 113: 1779-1784.

19 Sayegh RR, Ang LP, Foster CS, Dohlman CH. The Boston keratoprosthesis in Stevens-Johnson syndrome. Am J Ophthalmol 2008; 145: 438-444.

20 Akpek EK, Harissi-Dagher M, Petraca R, Butrus SI, Pineda 2nd R, Aquavella JV et al. Outcomes of Boston keratoprosthesis in aniridia: a retrospective multicentre study. Am J Ophthalmol 2007; 144: 227-231.

21 Khan BF, Harissi-Dagher M, Pavan-Langston D, Aquavella $\mathrm{JV}$, Dohlman CH. The Boston keratoprosthesis in herpetic keratitis. Acta Ophthalmol 2007; 125: 745-749.

22 Harissi-Dagher M, Dohlman CH. The Boston keratoprosthesis in severe ocular trauma. Can J Ophthalmol 2008; 43: 165-169.

23 Bradley JC, Hernandez EG, Schwab IR, Mannis MJ. Boston type I keratoprosthesis: the University of California Davis Experience. Cornea 2009; 28: 321-327.

24 Aldave AJ, Kamal KM, Vo RC, Yu F. The Boston type I keratoprosthesis: improving outcomes and expanding indications. Ophthalmology 2009; 116: 640-651.

25 Chew HF, Ayres BD, Hammersmith KM, Rapuano CJ, Laibson PR, Myers JS et al. Boston keratoprosthesis outcomes and complications. Cornea 2009; 28: 989-996.
26 Dunlap K, Chak G, Aquavella JV, Myrowitz E, Utine CA, Akpek E. Short-term visual outcomes of Boston type 1 keratoprosthesis implantation. Ophthalmology 2010; 117: 687-692.

27 Ciolino JB, Ament JW, Zerbe BL, Belin MW. Etiology of keratoprosthesis loss: results from the Boston Keratoprosthesis Multicenter Study. Invest Ophthalmol Vis Sci 2008; 49: E-Abstract 5712.

28 Aquavella JV, Qian Y, McCormick GJ, Palakuru JR. Keratoprosthesis: the Dohlman-Doane device. Am J Ophthalmol 2005; 140: 1032-1038.

29 Chak G, Aquavella JV. A safe Nd:YAG retroprosthetic membrane removal technique for keratoprosthesis. Cornea 2010; 29: 1169-1172.

30 Todani A, Ciolino JB, Ament JD, Colby KA, Pineda R, Belin MW et al. Titanium back plate for a PMMA keratoprosthesis:clinical outcomes. Greafes Arch Clin Exp Ophthalmol 2011; 249: 1515-1518.

31 Ament JD, Spurr-Michaud S, Dohlman CH, Gipson IK. The Boston keratoprosthesis: comparing corneal cell compatibility with titanium and PMMA. Cornea 2009; 28: 808-811.

32 Netland PA, Terada H, Dohlman CH. Glaucoma associated with keratoprosthesis. Ophthalmology 1998; 105: 751-757.

33 Ritterband DC, Shapiro D, Trubnik V, Marmor M, Meskin S, Seedor J et al. Penetrating keratoplasty with pars plana glaucoma drainage devices. Cornea 2007; 26: 1060-1066.

34 Kwon YH, Taylor JM, Hong S, Honkanen RA, Zimmerman $\mathrm{MB}$, Alward WL et al. Long-term results of eyes with penetrating keratoplasty and glaucoma drainage tube implant. Ophthalmology 2001; 108: 272-278.

35 Ray S, Khan BF, Dohlman CH, D'Amico DJ. Management of vitreoretinal complications in eyes with permanent keratoprosthesis. Arch Ophthalmol 2002; 120: 559-566.

36 Tay E, Utine CA, Akpek EK. Crescenteric amniotic membrane grafting in keratoprosthesis-associated corneal melt. Arch Ophthalmol 2011; 128: 779-782.

37 Barnes SD, Dohlman CH, Durand ML. Fungal colonization and infection in Boston keratoprosthesis. Cornea 2007; 26: 9-15. 